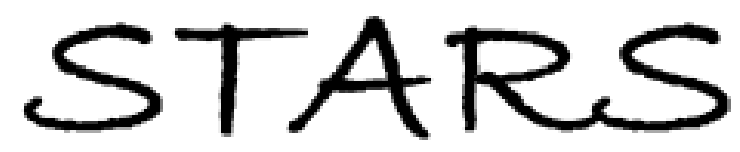

University of Central Florida

STARS

$1-1-2005$

\title{
Ion emission measurements and mirror erosion studies for extreme ultraviolet lithography
}

\author{
K. Takenoshita \\ University of Central Florida \\ C. -S. Koay \\ University of Central Florida \\ S. George \\ University of Central Florida \\ S. Teerawattansook \\ University of Central Florida \\ M. Richardson \\ University of Central Florida
}

Find similar works at: https://stars.library.ucf.edu/facultybib2000

University of Central Florida Libraries http://library.ucf.edu

See next page for additional authors

This Article; Proceedings Paper is brought to you for free and open access by the Faculty Bibliography at STARS. It has been accepted for inclusion in Faculty Bibliography 2000s by an authorized administrator of STARS. For more information, please contact STARS@ucf.edu.

\section{Recommended Citation}

Takenoshita, K.; Koay, C. -S.; George, S.; Teerawattansook, S.; Richardson, M.; and Bakshi, V., "Ion emission measurements and mirror erosion studies for extreme ultraviolet lithography" (2005). Faculty Bibliography 2000s. 5713.

https://stars.library.ucf.edu/facultybib2000/5713

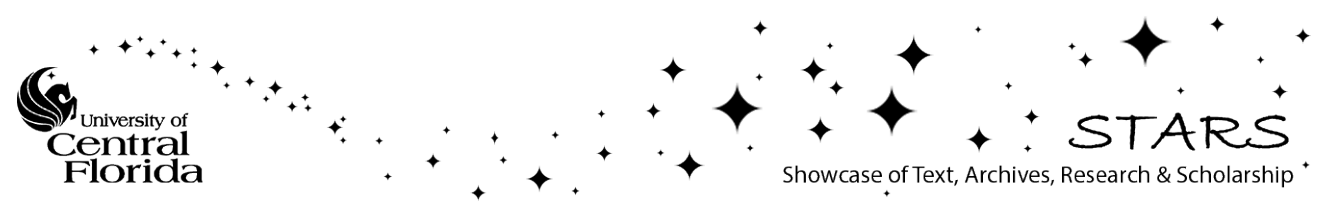




\section{Authors}

K. Takenoshita, C. -S. Koay, S. George, S. Teerawattansook, M. Richardson, and V. Bakshi 
Ion emission measurements and mirror erosion studies for extreme ultraviolet lithography

K. Takenoshita, C.-S. Koay, S. George, S. Teerawattansook, M. Richardson, and V. Bakshi

Citation: Journal of Vacuum Science \& Technology B: Microelectronics and Nanometer Structures Processing, Measurement, and Phenomena 23, 2879 (2005); doi: 10.1116/1.2131879

View online: https://doi.org/10.1116/1.2131879

View Table of Contents: https://avs.scitation.org/toc/jvn/23/6

Published by the American Institute of Physics 


\title{
Ion emission measurements and mirror erosion studies for extreme ultraviolet lithography
}

\author{
K. Takenoshita, C.-S. Koay, S. George, S. Teerawattansook, and M. Richardson \\ College of Optics \& Photonics: CREOL \& FPCE, University of Central Florida, \\ 4000 Central Florida Boulevard, Orlando, Florida 32816 \\ V. Bakshi \\ SEMATECH, Austin, Texas 78741
}

(Received 3 June 2005; accepted 10 October 2005; published 2 December 2005)

\begin{abstract}
Mirror erosion by high energy ion emission from extreme UV light sources is one of the main factors contributing to EUVL collector mirror reflectivity degradation. We are measuring ion energy distributions at the mirror distance from the plasma utilizing three different ion diagnostics for the case of tin-doped microscopic droplet laser plasmas. Typical ion energy distributions measured by an electrostatic spectrometer are described. From the ion energy distributions, an estimate of mirror erosion is obtained. The effectiveness of electrostatic field mitigation is evaluated for the EUVL source requirement. (C) 2005 American Vacuum Society. [DOI: 10.1116/1.2131879]
\end{abstract}

\section{INTRODUCTION}

Collector mirror lifetime is now one of the critical issues facing the successful introduction of extreme UV (EUV) lithography later this decade for computer chip manufacturing. Mirror erosion by high energy ion emission from EUV light sources is one of the main factors contributing to EUVL collector mirror reflectivity degradation. Erosion of multilayer mirrors by ion emission from laser plasma sources has been characterized with both water droplet ${ }^{1}$ and Xe gas jet target sources. ${ }^{2}$ Several studies indicate the very high energy ion emissions and erosions from Xe targets, ${ }^{3-5}$ despite this target material being initially selected for its potential to prevent mirror reflectivity degradation. Separately, we have introduced the use of tin as a target material, ${ }^{6}$ and we and others have made significant progress in improving conversion efficiency utilizing tin as the target material. ${ }^{7,8}$ Potentially, tin could be much more hazardous to mirror lifetime than Xe. Therefore, it is important to measure tin ion emission from the target in order to estimate its effect on mirror lifetime.

Ion emission from pulsed plasmas is often diagnosed with Faraday cup ion probes. These only register the ion current and give little information about the ion species emitted. Even with single-component plasmas, as for the Xe jet target, estimation of the ion spectrum from ion probes can be erroneous. To gain an understanding of the complete multiion energy distribution, particularly for multicomponent targets, it is necessary to use ion mass spectrometric techniques. We earlier reported the use of an ion spectrometer in combination with an ion probe, and showed that the ion probe signal consists of different ion species. ${ }^{9}$ This result also indicated the potential for integrated spectrometer measurements to provide a detailed ion energy distribution for each ion species. Quantitative analysis of the ion energy distributions can provide information about the energy and the number of ions hitting the mirror surface. This information can then be used to make a realistic prediction of the mirror reflectivity degradation lifetime by knowledge of the sputtering yield of conventional multilayer mirrors corresponding to the incident ion energy.

Our EUVL source approach utilizes the mass-limited target concept. ${ }^{10}$ The target contains just enough material components, that is, a known number of atoms, that are then converted into excited ions that radiate the requisite EUV radiation. The target is a microscopic droplet having a diameter encompassed within the laser focus so as to produce a nearly isotropic plasma. ${ }^{11}$ Comparison of the electron density profile of the expanding plasma with the predictions of a one-dimensional hydrocode simulation showed relatively good agreement. ${ }^{11}$ Inherently, the mass-limited target reduces to a minimum the rate of erosion, since the smallest amount of target material is used. Moreover, tight control of the irradiation and plasma coupling conditions can eliminate any generation of high energy (nonthermal) ions produced in the plasma. The challenge then is to prevent thermal ions from degrading mirror surfaces. We are currently examining the

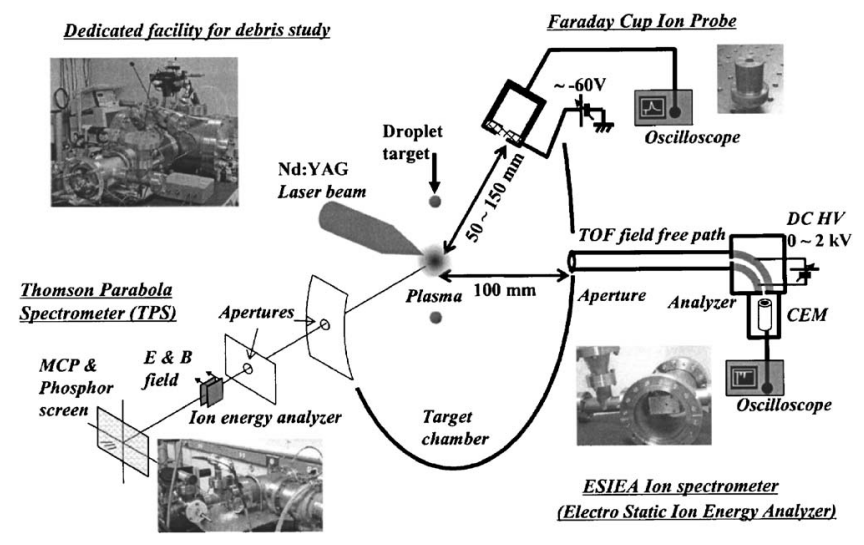

FIG. 1. Details of the dedicated droplet laser plasma facility with three ion diagnostics. 
use of electrostatic field mitigation (repeller field mitigation) $)^{1,7,9}$ to reduce the number of ions ablating the mirror surface.

Our studies focus on measuring the ions at the mirror distance, to provide details of ion energy distribution with which we can predict mirror lifetime. This article describes the following: (a) our experimental facility for the microscopic tin doped droplet EUV source, (b) some details of the ion energy distribution acquired with one of our ion spectrometers, (c) some estimates of mirror reflectivity degradation based on the ion energy distribution, and finally, (d) some measurements of the effectiveness of repeller field mitigation.

\section{DROPLET LASER PLASMA FACILITY DEDICATED TO ION MEASUREMENTS}

Our dedicated facility for ion emission measurements consists of a target chamber, laser system, droplet target dispenser, and ion diagnostics. The vacuum pressure in the target chamber is kept at $\sim 4 \times 10^{-4}$ Torr during the experiments. A commercial high repetition rate $\mathrm{Q}$-switched Nd:YAG laser is used for the plasma generation. The maximum laser pulse energy is $340 \mathrm{~mJ}$, the pulse duration is $\sim 10 \mathrm{~ns}$, and the repetition rate is $100 \mathrm{~Hz}$. The laser beam is focused onto the target using a lens with focal length of $50 \mathrm{~mm}$, which gives the maximum intensity of $\sim 6.8$ $\times 10^{11} \mathrm{~W} / \mathrm{cm}^{2}$ at the focal spot whose diameter is $70 \mu \mathrm{m}$. We use a multicomponent droplet target that includes tin atoms. The target containing 30\% tin water solution is delivered from a capillary nozzle. The number of tin atoms doped in a target is $\sim 10^{13}$ atoms. The $30-50 \mathrm{kHz}$ train of uniform droplets (from 30 to $50 \mu \mathrm{m}$ in diameter) is generated with a piezo-driven nozzle assembly and has a velocity $\sim 20 \mathrm{~m} / \mathrm{s}$. Unused targets are captured by a cryogenic cold trap in order to prevent evaporation in the vacuum chamber.

Three different approaches are used for ion measurement: a Faraday cup ion probe (IP), a custom-designed ion spectrometer configured as an electrostatic ion energy analyzer (ESIEA), and a custom-designed Thomson parabola ion spectrometer (TPS). These are shown in Fig. 1. The IP collects all charged particles unless an electrostatic potential is applied to the grid located in front of the cup electrode. To measure positively charged ions, a negative potential of $\sim 60 \mathrm{~V}$ is typically applied. At the beginning of the lasermaterial interaction, the IP experiences strong X-ray irradiation from the source, generating photoelectrons on the cup electrode. This photoelectron signal determines the plasma event time used for time-of-flight (TOF) ion measurements. From the TOF, and the distance between IP and the source, the velocity of the ions can be determined, assuming they have constant velocity. If the ion species that are producing the signal are known, then the ion kinetic energies can be calculated from the known mass of the ions.

While the IP collects a variety of ions, the ESIEA ion spectrometer detects selected ions with a corresponding kinetic energy. The spectrometer consists of an ion fluxlimiting aperture, a field-free path for TOF analysis, an en- ergy analyzer, and an ion detector. In our experiments, a $1 \mathrm{~mm}$ aperture selects a known fraction of the ion flux, and is placed at the distance of $10 \mathrm{~cm}$ from the source, equivalent to the distance of the first collector mirror in an EUVL source system. Behind the aperture, a separate vacuum chamber is attached to the target chamber as a field-free drift region for separating the ion species as they move at different velocities. The vacuum of the entire ion spectrometer assembly is kept at less than $1.0 \times 10^{-6}$ Torr. The geometry of the ESIEA consists of a set of two electrodes with the shape of a circular quadrant. Two $1 \mathrm{~mm}$ slits are placed at the entrance and the exit of the analyzer. By changing the applied potentials of the electrodes, one can selectively analyze the ions with the energies $(E)$ having different charge states $(Z)$ and mass $(M)$. The ion detector placed right after the analyzer detects those filtered ions with the same $E / Z$ and different TOF. A single-channel electron multiplier (CEM) is used for detecting the filtered ions. When the ions hit the surface of the CEM, secondary electrons are generated depending on the ion velocities. The efficiency of detecting an individual ion is a function of the ion velocity, as has been documented in earlier references. ${ }^{12-15}$ The efficiency has a steep cutoff in the lower ion energy region. From these references, it is reasonable to approximate the efficiency of the CEM as constant for energies higher than $1 \mathrm{keV}$. The distance from the plasma to the CEM is $900 \mathrm{~mm}$. Thus, a complete ion spectrum can be built by a making a sequence of ion measurements with the ESIEA set for different values of $E / Z$.

The TPS can resolve all ions species at one time. In this spectrometer, the ion stream through a small aperture is deflected by parallel electric and magnetic fields. The trajectory of each ion species traces out a unique parabolic shape orthogonal to the incident axis. A multichannel plate array coupled to a phosphor screen can then provide energy distributions for each value of $M / Z$. A comprehensive study for ion energy distributions based on TPS measurements is currently under way.

\section{SELECTED RESULTS FROM THE ESIEA SPECTROMETER}

A typical ESIEA spectrometer signal is shown in Fig. 2(a). Several peaks are observed in the signal because the different components of the target material arrive at the detector at different times depending on their mass charge ratio $(M / Z)$. These signal peaks are generated by those ions passing through the analyzer that hold the relationship expressed by the following equation:

$$
Z e E=\frac{m_{i} v_{i}^{2}}{R_{A}},
$$

where $Z$ is the ionization state of the ion species, $e$ is the unit charge, $E$ is the transverse electric field inside the analyzer in volts/meters, $m_{i}$ is ion mass in kilograms, $v_{i}$ is ion velocity in meters/second, and $R_{A}$ is the radius of the analyzer path in meters. The ion velocity is determined by the distance of 

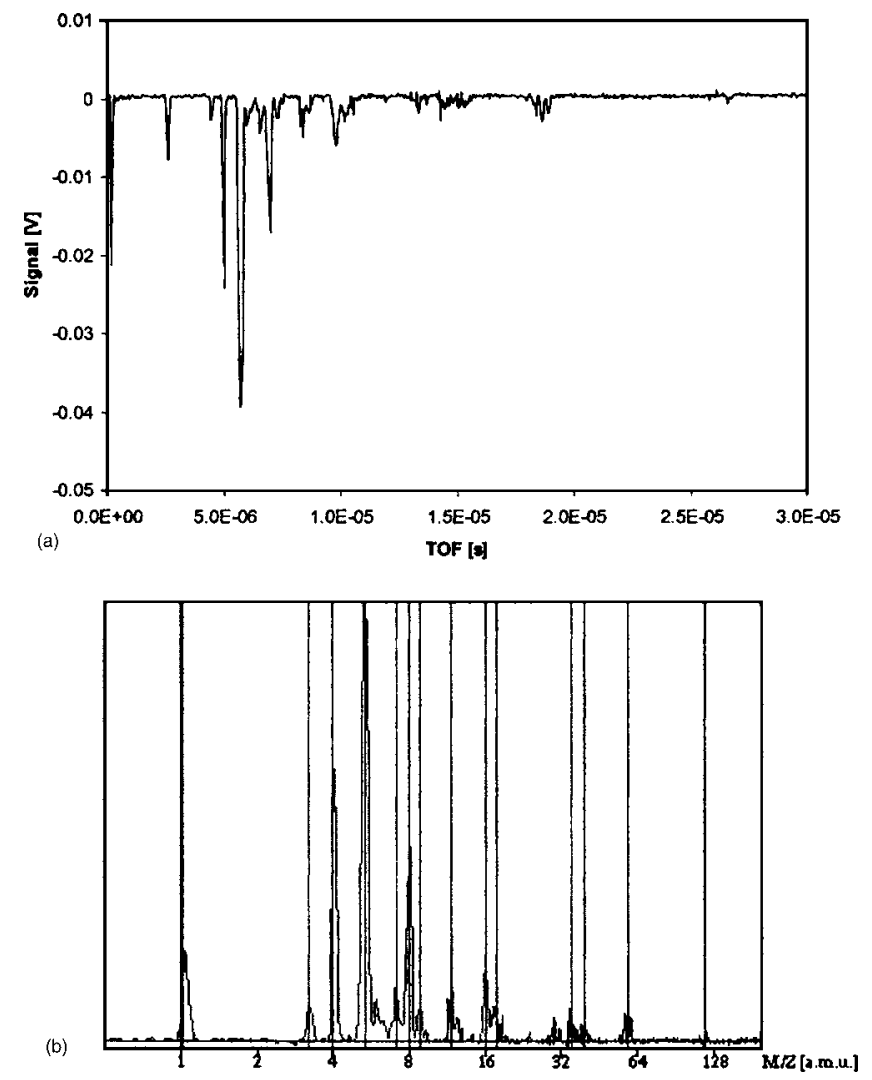

FIG. 2. (a) Typical ESIEA spectrometer signal. (b) Converted $M / Z$ signal.

CEM from the source and the TOF. Equation (1) is then reorganized in terms of $M / Z$, as

$$
\frac{M_{i}}{Z}=\frac{e R_{A} E}{m_{p} l^{2}}(\mathrm{TOF})^{2},
$$

where $M_{i}$ is the atomic weight of the ion of interest, $m_{p}$ is the proton mass in Kilograms, $l$ is the ion CEM distance from the plasma in meters, and TOF is the time-of-flight in the signal in seconds. To identify all the signal peaks, the signal is converted to a $M / Z$-based signal by using Eq. (2). A typical $M / Z$ signal is shown in Fig. 2(b). The $M / Z$ axis is plotted in logarithmic scale. All the peaks in the signal are identified as specific ion species.

By keeping the same plasma conditions, it is possible to obtain $M / Z$ signals with different energies by changing the electric field strength in the analyzer. It is then possible to plot all signals together and to investigate the signal profile for a constant $M / Z$, shown in Fig. 3(a). However, because different $M / Z$ spectra are converted from different TOF signals, the $M / Z$ values in all the signals plotted do not line up. A simple linear interpolation must be applied to the all converted signals. This complete map of $M / Z$ spectra at a constant plasma condition is shown in Fig. 3(b). It is possible to make profile for a constant $M / Z$, in other words, slicing the spectral map for each ion species can provide ion energy distribution.

To obtain the ion energy distributions for individual ion species, it is necessary to count the total charge from the
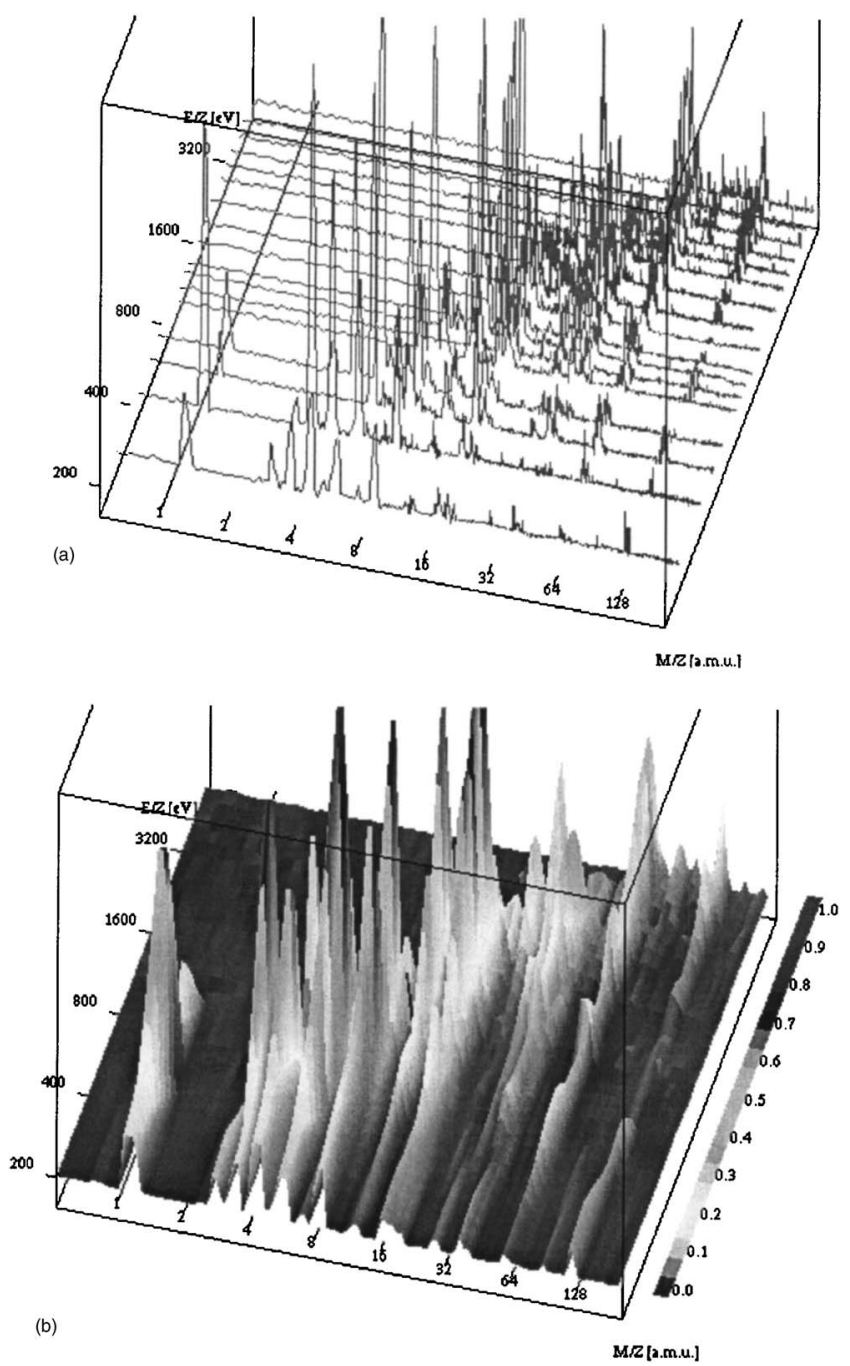

FIG. 3. (a) Series of $M / Z$ signals. (b) A map of $M / Z$ spectra.

CEM signal peak and to calculate the number of ions analyzed in the energy window of the analyzer. By obtaining the efficiencies of the ion-limiting aperture and the slit of the analyzer entrance, the distribution is calculated in terms of the number of ions per unit energy. The distribution is then estimated in terms of the unit solid angle, where isotropic ion emission is assumed. The details are described in the following.

To count the number of electrons in each CEM signal peak, it is necessary to specify the analyzer energy window and the corresponding TOF window. First, the nominal ion kinetic energy (KE) of the analyzer is expressed as

$$
\mathrm{KE}=\frac{1}{2} R_{A} Z e E,
$$

obtained from Eq. (1) by expressing $\frac{1}{2} m_{i} v_{i}^{2}=\mathrm{KE}$, where KE is expressed in joules. Because of the finite width of the slits of the analyzer, the analyzer has an energy window expressed by the equations 


$$
\begin{aligned}
& \Delta \mathrm{KE}=\mathrm{KE}_{\text {max }}-\mathrm{KE}_{\text {min }}, \\
& \mathrm{KE}_{\text {max }}=\frac{1}{2}\left(R_{A}+\frac{1}{2} \Delta R_{A}\right) Z e E, \\
& \mathrm{KE}_{\text {min }}=\frac{1}{2}\left(R_{A}-\frac{1}{2} \Delta R_{A}\right) Z e E,
\end{aligned}
$$

where $\Delta R_{A}$ is the width of the slit in meters. The corresponding TOF window is expressed in the following:

$$
\begin{aligned}
& \Delta \mathrm{TOF}=\mathrm{TOF}_{\min }-\mathrm{TOF}_{\max }, \\
& \mathrm{TOF}_{\max }=\frac{1}{l} \sqrt{\frac{M_{i} m_{p}}{2 \mathrm{KE}_{\max }}}, \\
& \mathrm{TOF}_{\min }=\frac{1}{l} \sqrt{\frac{M_{i} m_{p}}{2 \mathrm{KE}_{\min }} .}
\end{aligned}
$$

The signal integration can be calculated by multiplying the signal peak value and $\Delta$ TOF because the time constant of the CEM (see Ref. 15 ) is typically $\sim 4$ ns, much smaller than the acquisition time step of the signal. The number of electrons making the signal peak can now be calculated using

$$
-\frac{1}{e} \frac{V_{p}}{R_{t}} \Delta \mathrm{TOF},
$$

where $V_{p}$ is the signal peak in volts, $R_{t}$ is the terminal resistance of the oscilloscope in ohms. Because those electrons are multiplied by CEM based on the incident ions, the number of the incident ions $\Delta N_{i}$ is expressed as

$$
\Delta N_{i}=-\frac{1}{\eta} \frac{1}{G} \frac{1}{e} \frac{V_{p}}{R_{t}} \Delta \mathrm{TOF},
$$

where $\eta$ is the efficiency of CEM and $G$ is the gain of CEM. Here $\Delta N_{i}$ represents the number of ions analyzed in the energy window $\Delta \mathrm{KE}$. The ratio $\Delta N_{i} / \Delta \mathrm{KE}$ is approximated to the notation of the energy distribution $d N / d E$ when $\Delta N_{i}$ is approximately constant or $\Delta \mathrm{KE}$ is small. Finally, the ion energy distribution in terms of the number of ions per unit solid angle is obtained by calculating the efficiencies of the slit at the analyzer entrance and the aperture at known distances.

In the current experiments, $R_{A}$ is $25 \mathrm{~mm}$ and $\Delta R_{A}$ is $1 \mathrm{~mm}$, which determines $\Delta \mathrm{KE}$ to be $4.0 \%$ of nominal KE. $R_{t}$ is $50 \Omega$ and $G$ is $10^{6}$, which is a typical value. ${ }^{16} \eta$ is 0.8 , which is a reasonable assumption for tin over $1 \mathrm{keV}$. The collection ratio of the analyzer entrance slit is $9.8 \times 10^{-2}$ without considering ion beam divergence in the field-free path. The efficiency of the ion flux-limiting aperture is $6.3 \times 10^{-6}$.

Some estimated ion energy distributions of different ion species are shown in Fig. 4. Most of the ion emission detected is of low ionization states, typically less than $\mathrm{Sn}^{5+}$. These lower ionization states are observed at this distance from the plasma compared to those ionization states observed in the dense plasma source $\left(\mathrm{Sn}^{9+}-\mathrm{Sn}^{11+}\right)$ that contrib-
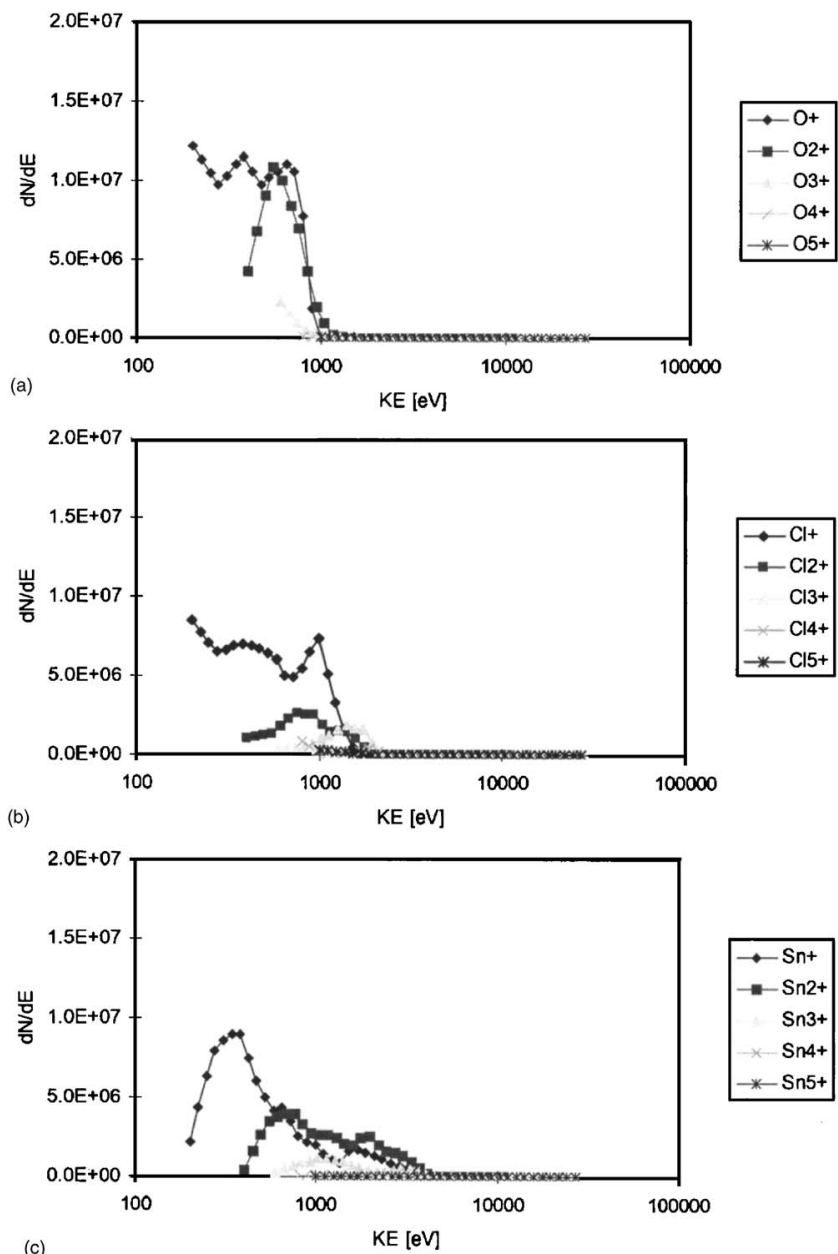

FIG. 4. Ion energy distribution for (a) oxygen, (b) for chlorine, and (c) for tin.

ute to the EUV radiation. These lower ionization states result from collisions and recombination occurring during plasma expansion.

\section{MIRROR SURFACE EROSION ESTIMATION}

Because the debris flux from the droplet laser plasma source is so low, and in addition, our laser irradiated droplet facilities operate at relatively low frequencies $(100 \mathrm{~Hz})$, we resort here to the use of numerical estimates of the sputtering yields, normalized against existing relevant experimental data. Unfortunately, we do not have reliable sputtering data from the tin-droplet laser plasma source. However, measured sputtering yields for $\mathrm{Xe}$ ions at energies in the range 0.5 $-5 \mathrm{keV}$ have been measured, ${ }^{17}$ and we utilize these to estimate the sputtering rate of a multilayer situated $20 \mathrm{~cm}$ from the tin-doped droplet source. The sputtering rates can be estimated using the Monte Carlo simulation, SRIM. ${ }^{18} \mathrm{We}$ assume that the sputtering rate of the mirror as a whole is largely that of the Si bilayers, and therefore use simply the sputtering yields for pure Si in our estimates, substituting the sputtering rate of $\mathrm{Si}$ for that of Mo. 

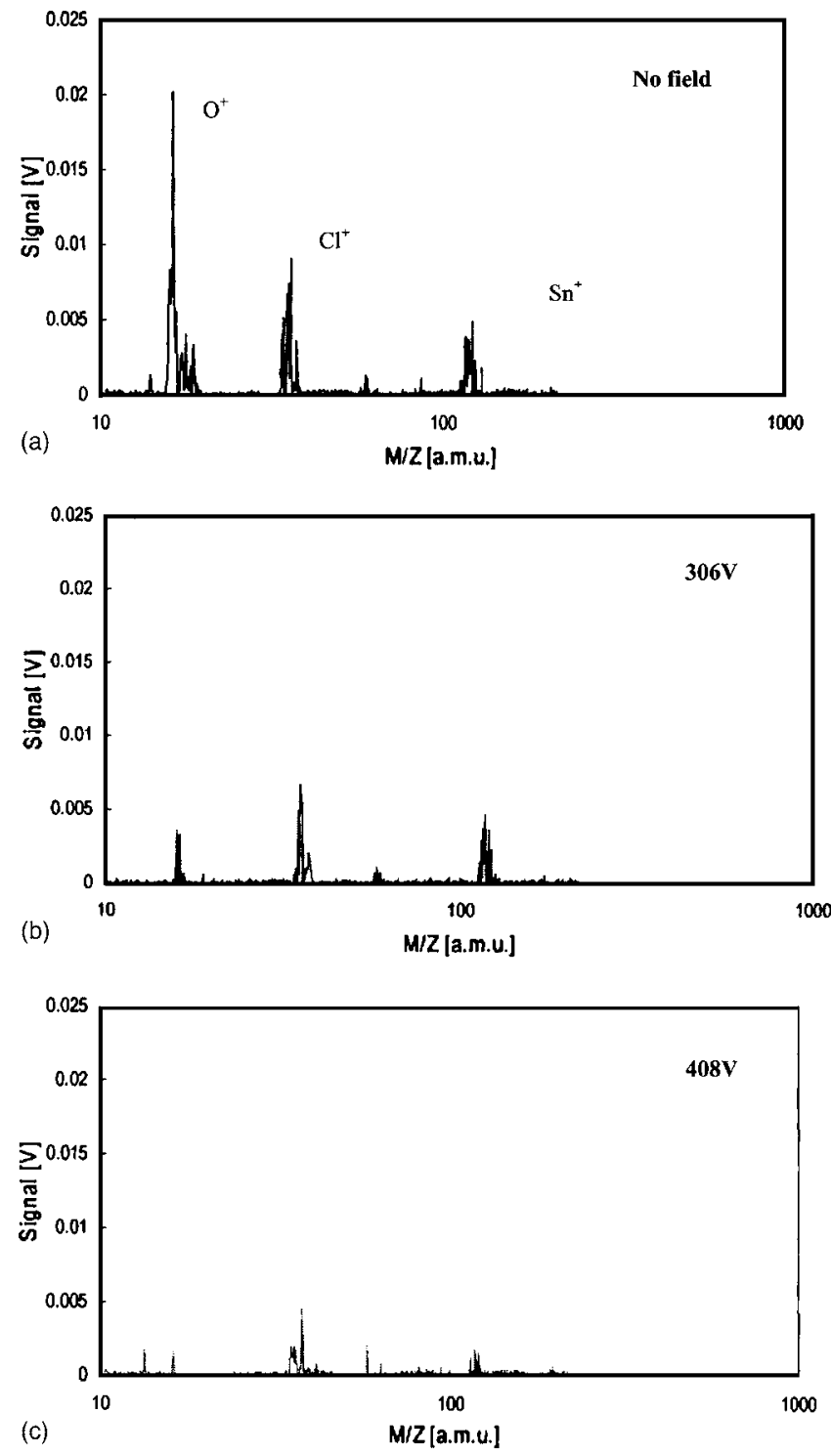

FIG. 5. (a) Ion spectra with no field applied, (b) with field potential of $306 \mathrm{~V}$, and (c) with $408 \mathrm{~V}$.

From the ion spectrometry results above, the predominate tin ions bombarding the mirror surface are measured to be $\mathrm{Sn}^{1+}, \mathrm{Sn}^{2+}$, and $\mathrm{Sn}^{3+}$ ions having energies in the range $1-3 \mathrm{keV}$ with a total flux of $1.5 \times 10^{10} \mathrm{ion} / \mathrm{sr}$. In the SRIM calculations of the resulting sputtering yield of these ions, we approximate their energy to be a triangular distribution between 1 and $3 \mathrm{keV}$, centered at $2 \mathrm{keV}$, with an even ion population among $\mathrm{Sn}^{1+}, \mathrm{Sn}^{2+}$, and $\mathrm{Sn}^{3+}$ ions. We now assume that the sputtering rate of $\mathrm{Si}$ for $\mathrm{Sn}$ ions of this energy is similar for Xe ions of the same energy. This is born out by comparative SRIM calculations for $\mathrm{Xe}$ and $\mathrm{Sn}$ ions of the same energy. Experimental data for $2-\mathrm{keV} \mathrm{Xe} \mathrm{ions}{ }^{17}$ indicates a sputtering rate of $\sim 5$ atoms/ion. We also assume, as the SRIM calculations indicate, that sputtering of Si by subkilovolt ions is negligible. The number of $\mathrm{Si}$ atoms in a volume of $1 \mathrm{~cm}^{2}$ by $1 \mathrm{~nm}$ thick is $5 \times 10^{15}$. With these considerations, we estimate the number of shots required to sputter a Si thickness of $1 \mathrm{~nm}$ to be $1.6 \times 10^{7} \mathrm{shots} / \mathrm{nm}$.
A typical $\mathrm{Mo} / \mathrm{Si}$ multilayer mirror comprises some 40 bilayers with a bilayer thickness of $6.9 \mathrm{~nm}$, and a fractional Mo composition of $40 \% .{ }^{19}$ If we assume a tolerable loss in reflectivity from a maximum of $70 \%$ to $\sim 60 \%$, this would imply an erosion of $\sim 20$ bilayers, having a total thickness of $138 \mathrm{~nm}$. The total number of shots needed to sputter this thickness of $\mathrm{Si}$ is $\sim 2.2 \times 10^{9}$, about one week $(\sim 170 \mathrm{~h})$ of target operation at a repetition rate of $10 \mathrm{kHz}$. The EUVL source requirement is for $30000 \mathrm{~h}$ continuous operation. Therefore, for the mass-limited target described here, an effective mitigation scheme or combination of schemes needs to reduce the ion flux by a factor of $\sim 200$. This is not a large number.

\section{ELECTROSTATIC FIELD MITIGATION}

We are currently evaluating the repeller field mitigation approach. In our present experiments we examine the effect of this scheme on the ion energy distribution that would be incident on the collection mirror at $10 \mathrm{~cm}$ distance from the source. The field is installed in front of the ion flux limiting aperture and the ESIEA measures ion signals for both cases of the field applied and with no field applied. The analyzed energy is set to $380 \mathrm{eV}$ so that singly charged oxygen ions, chlorine ions, and tin ions are detected. A typical result is shown in Fig. 5, showing that the peaks of the spectrometer signals for the ion species are reduced when the field potential approaches the kinetic energy of the ions where the initial field applied is $\sim 4 \mathrm{kV} / \mathrm{m}$. By counting the number of ions contributing to each ion signal peak, reduction ratios of about 200 for oxygen ions and 8 for tin ions are estimated. This result indicates that the reduction ratio for oxygen ions is sufficient with a repeller field alone but is not quite enough for tin. In addition high energy neutral atoms that are initially ionized and then recombined with electrons are not mitigated. It is possible to mitigate ions/neutrals while they are at higher ionization states. However, electrostatic fields will be shielded by the plasma where the Debye length is short, that is, where the electron density is high. Therefore, another mitigation scheme will need to be integrated with repeller field to satisfy the requirement.

\section{CONCLUSION}

This article describes the use of an ion spectrometer for making quantitative measurements of the ion emission from mass-limited droplet target laser plasmas. Detailed ion energy distributions, especially of tin ions, are obtained with this method, and a complete analysis of the ion energy distribution and its dependence on laser irradiation conditions will be made in a future publication. ${ }^{20}$ The result provides the energies and the number of ion emissions from the plasma source at the equivalent first mirror distance. This is essential for being able to estimate mirror erosion. Using the measured energy distributions, we have an estimate of the multilayer mirror lifetime for this particular source. The mirror lifetime under our current operating conditions is about two orders of magnitude shorter than the EUVL roadmap requires. We show that the repeller field mitigation scheme 
increases the mirror lifetime by about an order of magnitude. Further improvements will require perhaps the use of additional mitigation schemes.

\section{ACKNOWLEDGMENTS}

The authors wish to acknowledge the technical assistance of colleagues with LPL at the College of Optics \& Photonics: CREOL \& FPCE, particularly Dr. Greg Shimkaveg, Robert Bernath, and Joshua Duncan, and Zia Rahman and Kirk Scammon for assistance at the AMPAC/Material Characterization Facility. This work is supported by SEMATECH and the State of Florida.

${ }^{1}$ G. Schriever, M. Richardson, and I. C. E. Turcu, Proceedings of CLEO, 2000, pp. 393-394.

${ }^{2}$ G. D. Kubiak, EUVL Source Workshop, Dallas, Texas, 14 October 2002.

${ }^{3}$ R. J. Anderson, D. A. Buchenauer, L. Klebanoff, O. R. Wood II, and N. V. Edwards, Proc. SPIE 5374, 710 (2004).

${ }^{4}$ H. Komori et al., J. Vac. Sci. Technol. B 21, 2843 (2003).

${ }^{5}$ B. A. M. Hansson, O. Hemberg, and H. M. Hertz, Rev. Sci. Instrum. 75, 2122 (2004).

${ }^{6}$ E. Turcu, H. Rieger, M. Powers, M. Richardson, and C. Keyser, presented at Sematech Workshop on EUVL Source Development, Matsue, Japan,
2001.

${ }^{7}$ M. C. Richardson, C.-S. Koay, K. Takenoshita, and C. Keyser, J. Vac. Sci. Technol. B 22, 785 (2004).

${ }^{8}$ J. Pankert et al., Presentation at ISMT EUV Source Workshop, Antwerp, Belgium, 29 September 2003.

${ }^{9}$ K. Takenoshita, C.-S. Koay, S. Teerawattansook, and M. Richardson, Proc. SPIE 5374, 954 (2004).

${ }^{10}$ F. Jin, K. Gabel, M. C. Richardson, M. Kado, A. Vassiliev, and D. Salzmann, Proc. SPIE 2015, 151 (1994).

${ }^{11}$ C. Keyser, M. C. Richardson, and I. C. E. Turcu, Appl. Phys. A: Mater. Sci. Process. 77, 217 (2003).

${ }^{12}$ S. Shchemelinin, S. Pszona, G. Garty, A. Breskin, and R. Chechik, Nucl. Instrum. Methods Phys. Res. A 438, 447 (1999).

${ }^{13}$ M. Kremar et al., Appl. Radiat. Isot. 46, 477 (1995).

${ }^{14}$ I. S. Glimore and M. P. Seah, Appl. Surf. Sci. 144-145, 113 (1999).

${ }^{15}$ M. Krcmar et al., Radiat. Phys. Chem. 51, 437 (1998).

${ }^{16}$ Burle Technologies Inc., Channeltron-Electron Multiplier Handbook for Mass Spectrometry Applications available at http://www.burle.com/cgibin/byteserver.pl/pdf/ChannelBook.pdf

${ }^{17}$ G. Ecke, R. Kosiba, V. Kharlamov, Y. Trushin, and J. Pezoldt, Nucl. Instrum. Methods Phys. Res. B 196, 39 (2002).

${ }^{18}$ J. F. Ziegler, SRIM-2003, software available at http://www.srim.org/ SRIM/SRIMLEGL.htm.

${ }^{19} \mathrm{X}$-ray tools, Center for X-ray Optics, available at http://wwwcxro.lbl.gov/index.php?content $=/$ tools.html.

${ }^{20} \mathrm{~K}$. Takenoshita et al. (unpublished). 